\title{
ÉVALUATION DES PRÉDISPOSITIONS À L'ACTION SOCIALE
}

\author{
PATRICK O'NEILL et RACHEL THIBEAULT \\ Acadia University
}

RÉSUMÉ

La participation des citoyens aux changements sociaux peut dẻpendre, en partic, de certaines croyances que les individus ont au sujet du monde et de leur propre pouvoir à induire des changements. Afin d'évaluer la croyance en un pouvoir personnel et la croyance qu'il existe des injustices dans notre société, deux échelles ont été créées. La version anglaise de ces deux échelles a été validée par le procédé de Campbell et Fiske (1959) qui permet d'établir des niveaux de convergence et de différenciation. Les échelles ont prédit les résultats au cours d'expériences pertinentes et ont établi une nette distinction entre les groupes qui s'efforçaient de promouvoir des changements au sein de leur milieu et ceux qui demeuraient plutôt passifs. Cet article présente la version française de ces deux échelles ainsi que les données ayant trait a leur fidélité. Dans la premiére étude, nos données ont été recueillies auprès d'un échantillon d'une centaine d'Acadiens francophones habitant la NouvelleÉcosse. Les résultats indiquèrent que les échelles possèdent une validité de convergence et de différenciation. Dans la seconde étude, nous avons obtenu des données ayant trait à la stabilité des échelles en administrant les deux versions, la française et P'anglaise, à un échantillon bilingue, La corrélation entre les versions anglaise et française est tellement élevée qu'elles peuvent être considérées équivalentes.

Les minorités doivent se préparer à s'organiser si elles désirent protéger leurs droits et promouvoir leurs intérêts. Au Canada, ceci s'applique tout particulièrement aux minorités dont l'identité ethnique risque d'être assimilée au sein de la majorité. Cette situation se retrouve, par exemple, chez les francophones du Manitoba et de la Nouvelle-Écosse. Les efforts de la Société franco-manitobaine ont démontré à quel point une minorité organisée peut arriver à influencer le public et le gouvernement. D'autre part, la minorité acadienne néo-écossaise est

Les auteurs aimeraient remercier ceux qui ont contribue â ce projet, tout specialement Sheila Chandler et Lionel Thibeault, Nous desirons aussi exprimer notre reconnaissance à Maurice Payette pour ses commentaires des plus utiles au cours de la préparation initiale de cette recherche. Le conseil de recherches en sciences humaines du Canada a fourni le soutien financier nécessaire à ce travail et le gouvernement de la Nouvelle-Écosse a partiellement subventionné notre assistante de recherche, Les demandes de tirés-a-part doivent être adressées au Dr. Patrick O'Neill, Départenent de psychologie, Université Acadia, Wolfville, Nouvelle-Ecosse BOP IXO. 
vulnérable en temps de crise parce qu'elle ne possède pas une organisation aussi puissante. Durant les années 70 , les Acadiens habitant la région francophone de la Nouvelle-Écosse appelée French Shore furent outrés par l'ampleur de la résistance anglophone lorsqu'il fut question de déménager un escadron francophone à la base aérienne de l'endroit (O'Neill \& Levings, 1979). La réaction fut si forte que le député de comté prédit que les Acadiens se feraient molester. Plusieurs Acadiens réalisèrent alors à quel point les relations entre francophones et anglophones étaient fragiles au pays d'Évangéline. Il s'agit peut-être ici d'une leçon pour d'autres groupes minoritaires qui n'ont pas encore mis sur pied une organisation active et efficace pour les représenter. Étant donné que l'action sociale est importante dans le processus de protection des droits et de juste distribution du pouvoir et des ressources, il devient crucial d'étudier les facteurs qui la stimulent ou la restreignent.

\section{THÉORIE DE LA PARTICIPATION SOCIALE}

Pourquoi certains citoyens prennent-ils part à l'action sociale tandis que d'autres ne le font pas? Il est probable que les gens réagissent seulement contre ce qui menace leurs intérêts propres. On peut, par exemple, mobiliser plus facilement les gens pour s'opposer à un dépotoir de déchets radio-actifs si ce dernier est près de leur domicile. Mais cela ne constitue qu'une partie de la réponse. Les individus qui sont pareillement menacés par un problème social ne sont pas tous également motivés à changer la situation. Et, de plus, bien des gens sont passionnément engagés dans des causes qui n'affectent en rien leur propre bien-être; des femmes se vouent à aider les victimes de viol et d'agression conjugale même si elles n'ont jamais été personnellement confrontées à de telles situations.

Certains chercheurs proposent des explications psychologiques pour comprendre le phénomène de participation des citoyens. Payette (1983) a mis en évidence le fait que les projets sociaux doivent combler des besoins chez les participants bénévoles. Cook (1983) a constaté que, lorsque des citoyens s'identifient fortement à leur quartier, il est plus facile de les motiver à réagir quand ce quartier est menacé. Weick (1984) et Stokols (1975) ont cherché à comprendre comment les citoyens définissent un problème. La stratégie des petites victoires de Weick met l'accent sur une définition qui stipule que le citoyen ne doit pas se sentir dépassé par l'ampleur de la tâche à accomplir. Stokols a constaté que la quantité et la forme de participation dépendent toutes deux de la façon dont les citoyens perçoivent l'oppression (délibérée ou accidentelle) et leur marge de manoeuvre.

Notre propre recherche souligne deux catégories de croyances qui peuvent induire chez les individus une participation plus active à l'action sociale. Les gens devraient être enclins a s'engager s'ils croient que les conditions sociales sont souvent injustes, et qu'ils peuvent, personnellement, faire quelque chose contre de telles conditions.

Nous avions besoin d'échelles pour vérifier l'importance de ces catégories de croyances. Après avoir réalisé que les échelles existantes n'étaient pas adéquates pour notre usage (nos raisons sont décrites plus loin) nous avons développé deux nouvelles échelles. Nous nous sommes servis de la méthode de Campbell et Fiske (1959) pour établir la validité de la version anglaise et nous avons traduit ces 
echelles en français. Nous présenterons tout d'abord la théorie de participation sur laquelle $s^{\prime}$ appuient nos deux nouvelles échelles et nous expliquerons ensuite le travail effectué à l'aide de la version anglaise de ces mêmes échelles. Enfin, nous décrirons la version française ainsi que la fidélité obtenue dans nos deux études.

\section{L'injustice et le pouvoir personnel}

Afin d'amener les citoyens à s'impliquer dans l'action sociale, l'on doit vaincre ce que O'Neill et Trickett (1982) appellent la psychologie défaitiste. Cette psychologie s'appuie sur deux croyances fondamentales, la premiẻre étant que rien n'a besoin d'être changé et la seconde, qu'un citoyen n'a de toutes façons aucun pouvoir. La première croyance peut faire naftre chez un individu une certaine tendance à blâmer la victime, même s'il s'agit de lui. La deuxième croyance, elle, est reliée de près à la notion de contrôle externe (Rotter, 1966). Certains groupes de citoyens, plus que d'autres, semblent partager ces croyances. O'Neill et Loomes (1982) ont observé que l'obstacle majeur à surmonter lorsque l'on tente d'organiser un milieu défavorisé est souvent le sentiment d'impuissance face à l'oppression que ressentent ses membres; cependant, en milieu plus aisé, le problème se présente parfois différemment: on se heurte alors à la croyance que les gens n'obtiennent que ce qu'ils méritent et, vice-versa, méritent toujours ce qui leur arrive.

Selon Lerner et ses collègues (e.g., Lerner \& Miller, 1978; Lerner \& Simmons, 1966), les gens ont besoin de croire à un monde où ils obtiennent ce qu'ils méritent. Cette hypothèse d'un monde juste les rend capables de percevoir leur milieu comme étant stable et ordonné. Mais cette hypothèse les amène aussi à conclure que les gens méritent tout ce qui leur arrive. Goffman (1963) dit que l'on regarde souvent les infirmités comme la marque d'une tare morale; Ryan (1971) croit que la classe moyenne blâme les opprimés pour justifier la pauvreté et les effets du racisme. La tendance à dénigrer les victimes de désastres et ceux qui subissent les iniquités sociales ne disposera pas les citoyens a l'action sociale. Mais il est reconnu que les gens sont moins enclins à blamer les victimes s'il est en leur pouvoir de faire quelque chose pour rectifier la situation (Lincoln \& Levinger, 1972). Ce facteur nous amène donc à poser la question suivante: comment les citoyens perçoivent-ils leur capacité d'intervention en matière d'action sociale? C'est là qu'apparaît la notion de le pouvoir personnel.

Notre concept de croyance au pouvoir personnel est semblable à ce que deCharms (1968) appelle la causation personnelle, mais est plus limité que le concept de croyance au contrôle interne développé par Rotter (1966). Le pouvoir personnel repose sur la croyance de l'individu en son propre contrôle interne, contrôle qu'il n'étend pas nécessairement aux gens en géneral.

Un fort sens du contrôle interne est un pré-requis essentiel à l'action sociale. Pourquoi devrait-on s'impliquer si l'on ne s'attend pas à obtenir de résultats conerets? Les premiers travaux utilisant l'échelle Interne-Externe (I-E) de Rotter ont démontré que ceux qui avaient un fort sens du contrôle interne étaient plus souvent engagés dans l'action sociale (Gore \& Rotter, 1963; Strickland, 1965). Les études subséquentes n'ont pas confirmé ce rapport (Abramowitz, 1973, par exemple). L'aspect multidimensionnel de l'échelle en est peut-être la cause. 
Il est maintenant admis que les éléments de l'échelle I-E reliés à la vie même des répondants constituent un facteur bien distinct des éléments moins personnels qui font référence aux gens en général et à la société (Abrahamson, Schluderman, \& Schluderman, 1973; Abramowitz, 1973; Gurin, Gurin, Lao, \& Bettie, 1969; Mirels, 1970). Ce facteur personnel prédit l'accomplissement, la motivation et le niveau d'aspiration (Gurin et al., 1969; Lao, 1970) mais prédit rarement l'engagement social. Phipps-Sanger et Alker (1972) ont observé une relation semblable au sein du groupe féministe qu'ils étudiaient, mais d'autres recherches, effectuées auprès de groupes différents (Abramowitz, 1973; Gurin et al,, 1969; Lao, 1970) ont dẻmenti ce point de vue. Le fait que le pouvoir personnel prédise rarement l'engagement dans l'action sociale (Rotter, 1975) n'est pas surprenant. Il y a, peut-être, une autre croyance générale qui doive être associée au contrôle interne: la croyance que le monde puisse être injuste. Ả ce sujet, Lao (1970) rapporta quelques données intéressantes. Elle trouva que les étudiants noirs qui avaient la plus forte motivation à s'engager dans l'action contre le racisme étaient ceux qui blâmaient le système plutôt que les individus en tant que cause des problèmes sociaux. De plus, ils se révélaient d'orientation interne sur l'échelle I-E, et croyaient que la discrimination raciale pouvait être modifiée.

\section{Construction de deux échelles indépendantes}

De nouvelles échelles se sont avérées nécessaires pour étudier l'hypothèse que la participation des citoyens à l'action sociale est plus probable lorsque les citoyens croient que les conditions sociales sont souvent injustes et que leurs prises de position peuvent modifier considérablement ces conditions.

Au début, deux échelles qui existaient déjà furent considérés: l'échelle I-E (Rotter, 1966) et l'échelle du monde juste (Rubin \& Peplau, 1973, 1975). Bien que les échelles employées dans le cadre de cette recherche se soient inspirées de celles construites par Rotter et par Rubin et Peplau, elles n'ont pu être utilisées qu'après de profondes modifications. En outre, ces deux échelles ne pouvaient être utilisées pour évaluer des concepts foncièrement différents. En effet, le chevauchement entre les deux échelles originales est considérable et, de par leur structure, elles ne sont pas censées être indépendantes. Un énoncé de l'échelle du monde juste, par exemple, se lit comme suit: "Dans la plupart des métiers, ceux qui font bien leur travail atteignent les échelons supérieurs." Cette phrase indique une croyance au contrôle interne ainsi qu'une croyance en un monde juste. Un autre énoncé de l'échelle I-E affirme que: "Pour l'étudiant qui est bien préparé, il n'y a pas d'examens injustes," Cet énoncé semble reposer tout autant sur l'hypothèse d'un monde juste que sur le contrôle externe du renforcement.

Dans leur forme plus générale, les concepts de contrôle interne et d'un monde juste sont nécessairement reliés (O'Neill, 1981; O'Neill \& Trickett, 1982). L'individu d'orientation interne croit que les actions positives sont récompensées; c'est en même temps l'expression d'une croyance en un monde juste. L'individu qui soutient l'hypothèse d'un monde juste croit que les gens reçoivent ce qu'ils méritent (et méritent ce qu'ils reçoivent), et cette opinion présuppose que les gens exercent un certain pouvoir sur leurs propres récompenses et punitions. Le contrôle interne, tel qu'évalué par l'échelle I-E, prédit de façon fiable la croyance en un monde juste (Lerner \& Miller, 1978; Phares \& Wilson, 1972; Rubin \& Peplau, 1975). 
La relation entre le contrôle interne et la tendance à blâmer les victimes constitue un dilemme: en effet, il semble que pour s'engager dans l'action sociale, I'individu doive à la fois croire au contrôle interne et avoir tendance a blâmer les conditions sociales plutôt que les victimes. Néanmoins, plusieurs issues s'offrent à nous. L'une d'elles réside dans la distinction entre le fait de blâmer les victimes en tant que causes des problèmes sociaux et le fait de croire que les gens ont le pouvoir de résoudre ces problèmes. Une autre solution consisterait à prendre pour acquis que les citoyens ne généralisent pas leur propre sens du pouvoir personnel pour rejetter le blâme sur les autres.

Brickman et al. (1982) ont présenté quatre modèles qui s'appuient sur le fait qu'un individu doive parfois résoudre un problème qu'il n'a pas nécessairement crếé. Parmi ceux-ci, le modèle compensatoire ne rejette pas le blâme sur les victimes mais propose plutôt qu'il est en leur pouvoir de trouver des solutions. Ce modele est donc clairement le plus propice a l'action sociale. En théorie, il permettrait de modifier la corrélation entre le contrôle interne et la tendance à blâmer les victimes.

L'hypothèse que les gens perçoivent ces questions différemment selon qu'il s'agisse d'eux-mêmes ou qu'il s'agisse d'autrui pourrait peut-êtres constituer une autre solution à ce paradoxe. Les travaux concernant les facteurs de l'échelle I-E supportent cette idée. Il y a une différence entre les réponses des sujets s'ils répondent pour eux-mêmes ou pour les gens en général. Collins (1974) a établi que la croyance en un monde juste constituait un facteur bien distinct sur P'échelle I-E. Dix des onze énoncés reliés à la croyance en un monde juste ont été identifiés comme étant d'orientation interne et seulement deux de ces énoncés ont été formulés à la première personne. Si nous mettions l'accent sur le pouvoir personnel plutôt que sur l'internalité nous pourrions peut-être briser la relation entre l'internalité et la tendance à blâmer les victimes. Notre hypothèse de travail serait donc que la croyance au pouvoir personnel et a l'injustice sociale stimulent l'engagement social.

Pour mesurer les concepts de pouvoir personnel et d'un monde juste, nous avons entrepris de développer des échelles qui ne se chevauchaient pas. Pour l'échelle PP (pouvoir personnel), nous avons tout d'abord relevé les énoncés de l'échelle I-E qui étaient formulés à la première personne, et nous avons ensuite ajouté des énoncés qui nous apparaissaient semblables. Pour l'échelle Ij (injustice), nous avons aussi débuté en utilisant des énoncés de l'échelle du monde juste et en avons ajouté d'autres qui ne faisaient pas référence au pouvoir personnel. L'échelle de monde juste est formulée en termes extrêmes qui indiquent que le monde est soit juste ou soit injuste. Nous avons modifié cette approche afin d'inclure le concept d'une injustice possible mais pas nécessairement certaine (par exemple: "Nos tribunaux libèrent souvent les coupables tandis qu'ils condamnent des innocents").

\section{VERSION ANGLAISE DES ECHELLES}

Le procédé de Campbell et Fiske (1959) a été utilisé pour établir la validité de convergence et la validité de différenciation de la version anglaise des échelles $\mathrm{P}$ et Ij. Le terme convergence signifie qu'il y a corrélation entre une échelle et d'autres 
mesures des mêmes croyances; le terme différenciation s'applique lorsque deux échelles ne mesurent pas la me̊me croyance. Ce procédé exige que l'on mesure chacun des concepts en cause en utilisant au moins deux méthodes différentes. De plus, l'on doit mesurer au moins deux caractéristiques que l'on veut différencier en utilisant les mêmes méthodes générales. Des mesures différentes du concept devraient donner des résultats semblables pour établir la validité de convergence. Le concept dont il s'agit peut être différencié des autres concepts en utilisant les mêmes méthodes pour établir la validité de différenciation.

Afin d'établir la fidélité de la version anglaise, nous avons administré les échelles à 80 étudiants de niveau universitaire. Nous avons utilisé la méthode de Guttman (voir Selltiz, Wrightsman, \& Cook, 1977) pour établir la fidélité moitiémoitié de la version anglaise. Nous avons aussi effectué une corrélation entre le total des points obtenus pour chaque échelle. Ensemble, ces deux procédés nous ont fourni la convergence et la différenciation requises par la méthode de Campbell et Fiske (1959). Les résultats concernant la fidélité des travaux effectués avec la version anglaise des échelles sont présentés plus loin, afin de permettre une comparaison plus directe avec la version française.

Trois études ont été complétées, chacune d'elles augmentant la validité de la version anglaise des échelles (O'Neill, Duffy, Enman, Blackmer, Goodwin, \& Campbell, 1986). Nous commenterons brièvement ces études puisqu'elles constituent la toile de fond pour la présentation de la version française. Notre collègue, Charlene Duffy, a reproduit une expérience bien connue qui avait pour but la validation de l'échelle I-E (Rotter \& Mulry, 1965). Les sujets devaient déterminer le degré de similitude entre des angles donnés. L'expérimentatrice avait affirmé à la moitié d'entre eux que leur performance dépendant uniquement de leur niveau d'habileté alors que les instructions pour l'autre groupe stipulaient que tout était question de chance. Le temps requis par chaque sujet pour déterminer la similitude des angles a constitué la mesure du degré d'intérêt. Des scores élevés sur l'échelle PP ont prédit de façon fiable le niveau d'intérêt dans la condition expérimentale qui faisant appel à l'habileté des sujets. Comme prévu, l'échelle Ij n'a pas prédit les résultats. Ceci a donc fourni une certaine validité de convergence pour l'échelle PP ainsi qu'une validité de différenciation pour les deux échelles.

Dans le cadre de la deuxième expérience, Michael Enman a présenté à des sujets une histoire de cas décrivant un viol. Il leur a ensuite demandé d'indiquer à quel point ils considéraient la victime comme étant responsable de son propre malheur. Les sujets qui ont obtenu peu de points sur l'échelle Ij (indiquant par le fait même une tendance à adhérer au concept d'un monde juste) blâmaient plus la victime que ceux qui avaient obtenu un score plus élevé. Comme prévu, l'échelle P n'a pas prédit les résultats puisque l'expérience n'était pas théoriquement reliée à la croyance au pouvoir personnel. L'étude a donc fourni une validité de convergence pour l'échelle $\mathrm{Ij}$ ainsi qu'une validité de différenciation pour les deux échelles.

Dans la troisième étude, les échelles ont été administrées à trois groupes choisis au sein de la population en général et à un groupe d'étudiants universitaires. Deux des groupes oeuvrant en milieu communautaire étaient activement engagés à promouvoir des changements sociaux. Ils ont démontré les scores élevés prévus sur les échelles Ij et PP lorsqu'ils furent comparés à des mères célibataires 
qui n'étaient pas impliquées dans l'action sociale. Les étudiants universitaires ont marqué moins de points sur l'échelle d'injustice que les groupes d'activistes et ont obtenu plus de points sur l'échelle de pouvoir personnel que les mères célibataires. Ces dernières ont obtenu des scores élevés sur l'échelle $\mathrm{lj}$ mais très peu de points sur l'échelle PP. Notre hypothèse se révèle donc fondée: il y a corrélation entre l'engagement social et l'obtention de scores élevés sur les deux échelles.

\section{VERSION FRANÇAISE DES ÉCHELLES}

Le but de cet article est de présenter une version française des deux échelles ainsi que deux études de fidélité. Dans la première de ces études, les questionnaires ont été remplis par une centaine d'Acadiens francophones de NouvelleÉcosse. Dans la seconde, les versions anglaise et française des échelles ont été administrées à des sujets bilingues qui ont rempli les deux questionnaires à une semaine d'intervalle. La version française de ces échelles est présentée dans les tableaux 1 et 2 .

\section{TABLEAU 1}

\section{Les énoncés en l'échelle $\mathbf{P}$}

1. J'obtiens toujours de meilleurs résultats quand j'adopte une ligne de conduite définie que lorsque je m'en remets au hasard.

3. J'ai souvent l'impression que je pourrais tout aussi bien prendre mes décisions en jouant a pile ou face.

5. Quoi qu'il m'arrive, c'est à moi que je dois m'en prendre.

7. Jaime mieux ne pas planifier trop longtemps a l'avance parce que je ne peux habituellement pas prédire comment les choses vont tourner.

9. Mes malheurs résultent habituellement de mes erreurs.

11. Parfois je crois que je ne contrôle pas assez le cours de ma vie.

13. Si je planifie soigneusement, je suis certain que je peux atteindre mes buts.

15. Le hasard a joué un rôle important dans ma vie.

17. Si j'utilise la bonne approche, je peux persuader les gens de faire ce que je veux.

19. Bien souvent, j'ai l'impression que j'ai très peu d'influence sur les choses qui m'arrivent.

21. Les malheurs qui m'arrivent sont souvent dus à la malchance.

\section{TABLEAU 2}

\section{Les énoncés de I'échelle I}

2. Les gens reçoivent habituellement ce qu'ils méritent en ce bas monde.

4. Bien des gens souffrent sans l'avoir mérité.

6. Les gens qui se sentent bien seuls auraient do apprendre à s'entendre avec les autres.

8. Nos tribunaux liberent souvent les coupables tandis qu'ils condamnent les innocents.

10. Les gens qui souffrent de dépression sont trop sensibles aux événements.

12. Bien souvent les aspirants politiques qui prennent la défense de leurs principes n'arrivent pas à se faire elire.

14. Les gens qui sont prudents sont rarement victimes d'un crime.

16. Bien des automobilistes prudents meurent dans des accidents dont ils ne sont pas responsables.

18. Les qualités les plus admirables d'un enfant échappent bien souvent à ses parents. 
20. Les gens sont souvent accablés par une mauvaise réputation qu'ils n'ont pas méritée.

22. Les gens sont souvent victimes de crimes uniquement parce qu'ils sont au mauvais endroit au mauvais moment.

\section{Méthodologie}

Une centaine d'Acadiens francophones de la Nouvelle-Écose ont participé à notre étude. Une assistante de recherche acadienne, habitant la région francophone qui s'étend de Digby à Yarmouth, a administré les échelles en version française à tous les adultes de son village. Elle s'est ensuite déplacée vers l'est et vers l'ouest jusqu'à ce qu'elle obtienne un échantillon de 100 personnes. (Ceux qui connaissent la région se rappelleront sans doute que les maisons se situent tout le long du littoral.) L'assistante expliqua aux répondants que le but de l'étude était de valider la version française d'une échelle d'attitude préalablement développée en anglais. Les deux échelles furent administrées en même temps.

\section{Résultats}

Le but de notre étude était de nous assurer que la version française des échelles remplissait le critère de Campbell et Fìske (1959) qui se résume comme suit: la corrélation interne de chaque échelle devrait être plus forte que la corrélation avec des échelles évaluant une dimension différente. Afin d'établir la corrélation interne, nous avons utilisé la méthode moitié-moitié de Guttman. Pour l'échelle $\mathrm{Ij}$, le coefficient de corrélation entre les moitiés de la version française était de .69 . Ceci se compare avantageusement à la valeur de .56 obtenue préalablement avec la version anglaise. Pour l'échelle PP, le coefficient de corrélation était de .37 ce qui, encore une fois, est bien près de la valeur de .31 obtenue en utilisant la version anglaise. La corrélation inter-échelles a été calculée à Paide d'un coefficient de corrélation de Pearson. Si ces échelles mesuraient réellement des concepts différents, de faibles corrélations seraient obtenues entre les deux, et ce, à la fois en français et en anglais. La corrélation entre les échelles Ij et PP était de -.15 pour la version française, n'ayant donc aucune signification statistique. Un niveau de corrélation semblable $(-.07)$ avait été obtenu plus tôt en utilisant la version anglaise. Il est évident que la fidélité des échelles demeure la même en français et an anglais.

Puisque le développement d'échelles qui ne se chevauchent pas constitue un défi majeur dans ce domaine de recherche, il était important de démontrer que la corrélation inter-échelles était faible. En ce qui concerne la corrélation interne de l'échelle, il n'est pas surprenant de constater que la méthode moitié-moitié nous ait fourni une corrélation dont le coefficient est faible mais dont le seuil est significatif $(p<.05)$. Ce facteur semble lié au fait que chaque échelle ne comporte que onze énoncés couvrant des situations variées. Rotter (1975) fit remarquer que l'uniformité interne d'une échelle dépend largement de son contenu. Donc, si une échelle comprend des énoncés décrivant des situations très variées, son uniformité interne sera plus faible que si elle n'évaluait qu'un domaine très circonscrit. L'échelle $\mathrm{Ij}$, par exemple, renferme des énoncés ayant trait au système judiciaire, aux problèmes émotionnels, aux accidents de la route, à la politique et au rôle de parent, et ce, en l'espace de quelques questions seulement. Quoique nous ayons tenté de restreindre le champ de notre échelle PP en utilisant strictement des items 
formulés à la première personne, le contenu n'en demeure pas moins vaste, englobant les concepts de chance, de destin, d'optimisme, de prise de décision, de prévoyance et de contrôle.

Par surcroît, il semble préférable d'utiliser un procédé de stabilité pour établir la fidélité d'échelles de ce type. Cette méthode a été employée dans l'étude suivante au cours de laquelle des répondants bilingues ont rempli les questionnaires anglais et français à une semaine d'intervalle.

\section{Méthodologie}

Les questionnaires furent distribués à 30 répondants bilingues, pour la plupart des enseignants ou des étudiants à la maîtrise. Un des sujets n'a pas rempli correctement le questionnaire et fut éliminé de l'étude. Notre échantillon final comporte done 29 sujets. Dix-huit d'entre eux ont indiqué que le français était leur langue maternelle: 14 étaient originaires du Québec et quatre de France. Parmi les neuf sujets qui se considéraient anglophones, il y avait sept Canadiens, un Américain et un Anglais. On retrouve aussi dans notre échantillon un Palestinien dont la langue maternelle est l'arabe et une femme originaire des lles Seychelles qui a appris le créole, le français et l'anglais simultanément.

Le chercheur expliqua aux répondants que le but de l'étude consistait à valider la version française d'une échelle préalablement développée en anglais. Une enveloppe contenant les deux échelles fut remise à chaque participant. Les instructions stipulaient qu'ils devaient remplir le premier questionnaire en tenant compte de leurs attitudes réelles et d'attendre une semaine avant de compléter le second. Le deuxième questionnaire devait être rempli indépendamment du premier et le tout devait être ensuite posté aux auteurs. Dans la moitié des cas, la version française était placée sur le dessus.

\section{Résultats}

Un intervalle d'une semaine serait plutôt court pour évaluer la fidélité entre les deux versions si les échelles administrées étaient les mêmes. Mais, dans notre cas, ce laps de temps nous a semblé suffisant puisque nous utilisions des versions dans les deux langues qui d'ailleurs différaient légèrement dans leur formulation. Nous avons utilisé le coefficient de corrélation de Pearson pour comparer les deux versions. La corrélation entre les deux versions de l'échelle PP était de .85; la corrélation entre les deux versions de l'échelle I était de .73. Les deux corrélations étaient statistiquement significatives à $p<.001$.

Nous sommes satisfaits de l'ampleur des corrélations étant donné que certains facteurs auraient pu diminuer nos résultats. Le caractère parfois imprécis de la traduction, par exemple, aurait pu affecter la réaction des répondants. Du reste, comme deux de nos sujets l'ont affirmé, il est possible que les gens voient le monde sous un jour différent selon qu'ils s'expriment en anglais ou en français. Bien qu'aucun de ces facteurs n'ait présenté de difficultés, nous avons néanmoins observé que la corrélation entre les deux échelles semble avoir été légèrement diminuée par une connaissance imparfaite de la langue seconde, surtout au niveau des expressions idiomatiques. Nous avons demandé à de nombreux sujets de relever tout ce qu'ils considéraient contradictoire dans les deux versions et nous avons découvert que certains ne saisissaient pas les nuances du texte rédigé dans la 
langue seconde. Une Française, par exemple, répondit oui à l'énoncé "les qualités les plus admirables d'un enfant échappent bien souvent à ses parents" alors qu'elle répondit non à l'énoncé anglais: "Parents often overlook the most admirable qualities in their children." Par la suite, elle expliqua qu'elle avait traduit littéralement le mot "overlook" lui donnant alors le sens de "surveiller" plutôt que celui, très différent et seul acceptable, de "négliger." Il nous est apparu que moins un sujet maîtrise les idiotismes de la langue seconde, plus la corrélation entre les échelles est faible. Malgré tout, les corrélations entre les versions anglaise et française sont assez élevées pour nous permettre de considérer les deux échelles comme étant équivalentes.

\section{DISCUSSION GÉNÉRALE}

Le but de notre recherche actuelle est de développer des instruments qui nous permettront d'identifier les croyances qui stimulent ou qui tempèrent la participation des citoyens. Les échelles PP et $\mathrm{Ij}$ ne sont pas destinées à l'usage des organisateurs en milieu communautaire; elles ont été plutôt créées dans le but d'aider les chercheurs a étudier les multiples aspects de la participation des citoyens. Est-il important, par exemple, d'utiliser des arguments différents pour mobiliser des classes différentes? Les organisateurs de milieux défavorisés prétendent qu'un sentiment d'impuissance doit presque toujours y être surmonté. Kahn (1970) affirme qu'il est essentiel que la première tentative d'un groupe soit couronnée de succès pour contrebalancer l'attitude défaitiste et fataliste que partagent fréquemment les membres d'une communauté pauvre: ils croient que rien ne peut améliorer leurs conditions de vie. Il souligne que si la première démarche se solde par un échec, l'attitude défaitiste s'en trouve renforcée. D'autre part, en ce qui concerne la classe moyenne, l'obstacle principal réside dans la croyance en un monde juste où la tendance à blâmer les victimes semble justifiée. $O^{\prime}$ Neill et Loomes (1982) ont décrit la création d'une organisation qui avait pour but Pobtention de services plus nombreux pour les enfants qui présentaient des difficultés d'apprentissage. Les membres de cet organisme, surtout des parents, appartenaient de manière prédominante à la classe moyenne. Ils étaient déjà conscients de leur pouvoir à induire des changements mais devaient tout de même réaliser que le gouvernement réagit beaucoup plus lentement envers les minorités qu'ils ne l'avaient cru.

Les organisateurs qui n'arrivent pas à identifier et à utiliser les croyances en cause risquent l'échec. Une anecdote d'Alinsky (1946) illustre bien les dangers d'un tel malentendu. Des organisateurs se présentèrent dans une ville dirigée par une grande compagnie et essayèrent d'enrôler les gens en leur disant que la compagnie était la source de tous leurs maux. Ils furent déçus de constater que très peu de résidents assistèrent à la réunion initiale. Alinsky suggère que les gens connaissaient déjà la cause de leurs problèmes ... "Ce gars-là, il est pas d'ici et il a l'air intelligent mais il doit me trouver borné-ça fait 40 ans que je reste dans la misère et il a le front de venir me dire pourquoi j'en arrache ... il pense peut-être que je suis même trop borné pour savoir ça." Ce que les gens voulaient savoir ce n'était pas qui avait causé leurs problèmes mais plutôt comment ils pouvaient les résoudre. 
Il est aussi possible que différentes croyances soient reliées à différents modes de participation des citoyens. Dans cet article, nous avons mis l'accent sur P'engagement dans l'action sociale. Mais, comme Payette (1983) le fit remarquer, il y a divers types de participation. Un citoyen peut avoir l'opportunité de travailler à l'amélioration de son quartier ou d'aider ceux qui sont moins fortunés sans pour autant percevoir ses actions comme une contribution significative pour changer le système et ses injustices (M. Payette, correspondance personnelle, 14 janvier, 1985). Il est probablement important que les militants croient que leurs actions sont efficaces sans qu'ils soient pour autant sensibles à l'injustice sociale.

Notre recherche actuelle indique qu'il existe une association entre des scores élevés sur les échelles Ij et PP et la participation des citoyens à l'action sociale. Les gens qui obtiennent un score élevé sur une des échelles et peu de points sur l'autre possèdent peut-être aussi des caractéristiques qui leur sont propres. Un individu ayant un score élevé sur l'échelle $1 \mathrm{j}$ mais bas sur l'échelle PP est peut-être plus motivé à promouvoir ses intérêts personnels qu'à s'impliquer dans l'action sociale. Par ailleurs, un individu dont le score est plus élevé sur l'échelle lj que sur l'échelle PP se sent peut-être aliéné par une injustice sociale qu'il sernble impuissant à redresser. Ces questions pourraient être abordées lors de recherches futures.

La version française des deux échelles que nous avons décrites est destinée à aider les chercheurs à étudier le phénomène de la participation des citoyens. Nous nous attendons à ce que ces échelles se révèlent tout aussi utiles en tant qu'outils de recherche que celles que nous venons de mentionner. Le travail de validation se déroulera de la même manière que celui qui a été fait avec la version anglaise. Étant donné que les travaux effectués avec la version anglaise semblaient des plus prometteurs, il nous a paru opportun de présenter à la fois la traduction et les données concernant la fidélité afin que le tout soit disponible pour les chercheurs travaillant en français.

\begin{abstract}
Citizen participation in social change may depend, in part, on certain beliefs about the world and about the power of the citizen to change social conditions. To evaluate the belief in personal power and the belief that social conditions are often unjust, two scales were created. The multi-trait multimethod matrix of Campbell and Fiske was used to establish convergent and divergent validity of the English version of the scales. The scales predicted results in relevant experiments and distinguished among community groups that were, or were not, involved in social action. This article presents French versions of the two scales and reliability data from two studies. In the first study, data were gathered from a sample of 100 Acadians in Nova Scotia. The results indicated that the scales have both convergent and divergent validity. In the second study, we administered both the English and the French versions of the scales to a bilingual sample. The correlations between the English and French versions were sufficiently high for the versions to be considered equivalent.
\end{abstract}




\section{RÉFÉRENCES}

Abrahamson, D., Schludermann, S., \& Schludermann, E. (1973), Replication of dimensions of locus of control. Journal of Consulting and Clinical Psychology, 41, 320.

Abramowitz, S.I. (1973). Internal-external control and social-political activism: A test of the dimensionality of Rotter's Internal-External Scale. Journal of Consulting and Clinical Psychology, 40, 196-201.

Alinsky, S.D. (1946). Reveille for radicals. New York: Random House.

Brickman, P., Rabinowitz, V.C., Karuza, J., Jr,, Coates, D., Cohn, E., \& Kidder, L. (1982). Models of helping and coping. American Psychologist, 37, 368-384.

Campbell, D.T., \& Fiske, D.W. (1959). Convergent and discriminant validation by the multitrait-multimethod matrix. Psychological Bulletin, 56. 81-105.

Collins, B.E. (1974). Four components of the Rotter Internal-External Scale: Belief in a difficult world, a just world, a predictable world, and a politically responsive world. Journal of Personality and Social Psychology, 29, 381-391.

Cook, J.R. (1983). Citizen response in a neighborhood under threat. American Journal of Community Psychology, 11, 459-471.

deCharms, R. (1968). Personal catsation. New York: Academic Press.

Goffman, E. (1963). Notes on the management of spoiled identity. Englewood Cliffs, N.J.: Prentice-Hall.

Gore, P.M., \& Rotter, J.B. (1963). A personality correlate of social action. Journal of Personality, 31, 58-64.

Gurin, P., Gurin, G., Lao, P.C., \& Beattie, M. (1969). Internal-external control in the motivation dynamics of Negro youth. Journal of Social Issues, 25, 29-53.

Kahn, S. (1970). How people get power. New York: McGraw-Hill.

Lao, R.C. (1970). Internal-external control and competent and innovative behavior among Negro college students. Journal of Personality and Social Psychology, 14, 263-270.

Lerner, M.J., \& Miller, D.T. (1978). Just world research and the attribution process: Looking back and looking ahead. Psychological Bulletin, 85, 1030-1051.

Lerner, M.J., \& Simmons, C.H. (1966). Observer's reaction to the "innocent victim": Compassion or rejection? Journal of Personality and Social Psychology, 4, 203-210.

Lincoln, A., \& Levinger, G. (1972). Observer's evaluations of the victim and the attacker in an aggressive incident. Journal of Personality and Social Psychology, 22, 202-210.

Mirels, H.L. (1970). Dimensions of internal versus external control. Journal of Consulting and Clinical Psychology, 34, 226-228.

O'Neill, P. (1981). Cognitive community psychology. American Psychologist, 36, 457-469.

O'Neill, P., Duffy, C.M., Enman, M.G., Blackmer, E., Goodwin, J.P., \& Campbell, R.E. (1986). Cognition and citizen participation in social action. Manuscrit soumis pour publication.

O'Neill, P., \& Levings, D.E. (1979). Inducing biased scanning in a group setting to change attitudes toward bilingualism and capital punishment. Journal of Personality and Sacial Psychology, 37, 1432-1438.

O'Neill, P., \& Loomes, P.R. (1982). Building a community group to improve local schools, In J.L. Alpert \& associates (Eds.), Psychological consultation in educational settings (pp. 275-303). San Francisco: Jossey-Bass.

O'Neill, P., \& Trickett, E.J. (1982). Community consultation. San Francisco: Jossey-Bass.

Payette, M. (1983), La participation communautaire comme réponse A des besoins psychologiques. La Revue Canadienne de Sante Mentale Communautaire, 2, 21-30.

Phares, E.J., \& Wilson, K.G. (1972). Responsibility attribution: Role of outcome severity, situational ambiguity, and internal-external control. Journal of Personality, 40 , 392-406.

Phipps-Sanger, S., \& Alker, H.A. (1972). Dimensions of internal-external locus of control and the Women's Liberation movement. Journal of Social Issues, 28, 115-129. 
Rotter, J.B. (1966). Generalized expectancies for internal versus external control of reinforcement. Psychological Monographs, 80 (1, Whole No. 609).

Rotter, J.B. (1975). Some problems and misconceptions related to the construct of internal versus external control of reinforcement. Journal of Consulting and Clinical Psychology, 43, 56-67.

Rotter, J.B., \& Mulry, R.C. (1965). Internal versus external control of reinforcement and decision time. Journal of Personality and Social Psychology, 2, 598-604.

Rubin, Z., \& Peplau, A. (1973). Belief in a just world and reactions to another's lot: a study of participation in the National Draft Lottery. Journal of Social Issues, 29, 73-93.

Rubin, Z., \& Peplau, A. (1975). Who believes in a just world? Journal of Social Issues, 31, 65-89.

Ryan, W. (1971). Blaming the victim. New York: Pantheon Books.

Selltiz, C., Wrightsman, L.S., \& Cook, S.W. (1977). Les méthodes de recherche en sciences sociales (D. Bélanger, Trans.). Montréal: Les Éditions HRW Ltée.

Stokols, D. (1975). Toward a psychological theory of alienation. Psychological Review, 82, 26-44.

Strickland, B.R. (1965). Prediction of social action from a dimension of internal-external control. Journal of Social Psychology, 66, 353-358.

Weick, K.E. (1984). Small wins: Redefining the scale of social problems. American Psychologist, $39,40-49$. 